\title{
ENGENHARIA ASSISTIDA POR COMPUTADOR (CAD-CAE): IMPLEMENTAÇÃO NA GRADE CURRICULAR DE CURSO DE ENGENHARIA NO BRASIL
}

\author{
IVANDO S. DinIZ1 ${ }^{1}$, WeSLEY A. SOUZA ${ }^{1}$, LUIZ C. S. GÓES ${ }^{2}$ \\ 1. Universidade Estadual Paulista "Júlio de Mesquita Filho" \\ Avenida três de março, 511, alto da boa vista. Sorocaba. SP - CEP 18087-180 \\ E-mails: ivando@sorocaba.unesp.br, wesley@fc.unesp.br \\ 2. ITA - Instituto Tecnológico de Aeronáutica \\ Praça Marechal Eduardo Gomes, 50, Vila das Acácias. São José dos Campos. SP - CEP 12.228-900 \\ E-mail: goeseita.br
}

\begin{abstract}
This paper presents resources to motivate universities to include computational tools, particularly in the teaching of modeling (design) of dynamical assisted systems (CAD-CAE) in undergraduate courses. The engineering in Brazil have several features for mathematical representation of dynamical systems via differential equations but these resources are applied to mathematical problems merely didactic. As the problem becomes more complex, the solution becomes impossible, except in the case of postgraduate education (Masters or $\mathrm{PhD}$ ), however, the modeling time in the thesis becomes unviable for the industry. The computer-aided tools are solved engineering problems in complex projects with excellent results. View the great importance of this area, the universities of Brazil still provide limited resources for computer-aided design teaching, among the reasons has been the absence of financial resources and also because not be contained in the curricular program. Thus, in this paper, we show some results of computer-aided models and present the importance of preparing future engineers in this area so that the industries have qualified manpower in order to increase the speed in obtaining the solutions. Projects of national industries, for the most part, are imported from matrix and the Brazil needs to emphasize this teaching methodology, thus seeking greater technological growth.
\end{abstract}

Keywords — Dynamic Systems Application, CAD-CAE, Modeling Sytems, Computer-Aided Design.

Resumo - Este trabalho apresenta recursos para motivar as universidades a incluírem ferramentas computacionais, sobretudo no ensino de modelagem (projeto) de sistemas dinâmicos assistido por computador (CAD-CAE) nos cursos de graduação. As engenharias do Brasil dispõem de vários recursos matemáticos para representação de sistemas dinâmicos através de equações diferenciais, mas estes recursos matemáticos são aplicados a problemas meramente didáticos. À medida que o problema fica mais complexo, a solução se torna impossível, salvo em caso do ensino de pós-graduação (mestrado ou doutorado), todavia, o tempo de modelagem na tese torna-se inviável para a indústria. As ferramentas de engenharia assistidas por computador têm solucionado problemas em projetos de grande complexidade com excelentes resultados. Vista a grande importância desta área, as universidades do Brasil ainda oferecem poucos recursos de ensino em projeto assistidos por computador, entre os motivos tem-se a falta de recursos financeiros e também por não estar contido na grade curricular. Desta forma, neste trabalho, procuramos mostrar alguns resultados de modelos assistidos por computador e apresentar a grande importância da preparação do futuro engenheiro nesta área para que as indústrias tenham mão de obra qualificada, com o intuito de aumentar a velocidade na obtenção das soluções. Os projetos das indústrias nacionais, em sua maioria, são importados da matriz e o Brasil precisa dar ênfase a esta metodologia de ensino, buscando assim maior crescimento tecnológico.

Palavras-chave - Aplicação de sistemas dinâmicos, CAD-CAE, Modelagem, projetos assistidos por computador.

\section{Introdução}

Os recursos computacionais estão conquistando espaços cada vez maiores nas diversas atividades de engenharia. Estes recursos, impulsionados pela constante evolução computacional em termos de hardware e software, permitem o desenvolvimento de poderosas ferramentas de projetos de engenharia.

Uma dessas ferramentas é conhecida como CAE (Computer Aided Engineering), ou engenharia auxiliada por computador, que consiste na utilização de recursos computacionais para simulação de modelos físicos, englobando a validação, simulação e otimização dos mesmos. Quando comparados aos protótipos clássicos, os modelos CAE apresentam maior versatilidade, proporcionando assim significativa redução de tempo e de custos.
A modelagem geométrica do produto utilizando um sistema de CAD (Computer Aided Design) é o primeiro passo da sequência de desenvolvimento de produtos com sistemas computadorizados. A informação gerada pelo sistema CAD, pode posteriormente ser exportada em formato padrão (IGES, STL, VDA, STEP, etc.) para o sistema CAE, permitindo assim, simular numericamente o modelo e/ou para um sistema CAM (Nakamura, et al., 2003).

A Figura 1 ilustra, de forma genérica, a integração dos softwares. Observar-se que o CAD é o centro dessa integração, ou seja, a base de todo o processo: CAE, CAM e CAPP.

No curso de graduação, diversas disciplinas voltadas a ao projeto assistido por computador poderiam estar ou acrescentada a grade curricular como parte do projeto pedagógico de cursos de engenharia do Brasil (Schvarcz et. al., 2010). Disciplinas como estática, resistência dos materiais, dinâmica de siste- 
mas, elementos de máquinas, projeto de mecanismo, introdução à teoria de controle, mecânica dos fluídos, transferência de calor e massa, entre outras dezenas poderiam ter aulas práticas assistidas por computador.

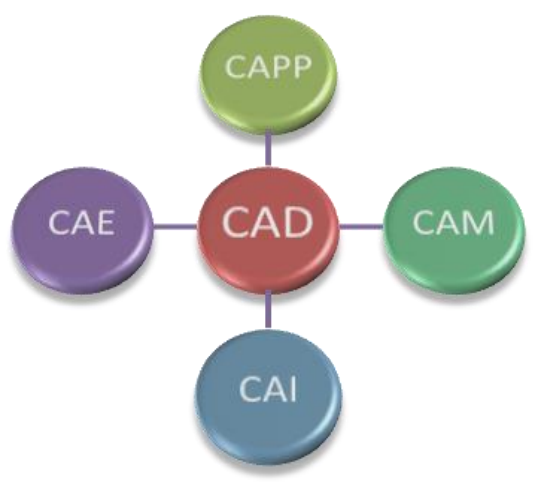

Figura 1. Integração dos softwares de engenharia auxiliada por computador.

O conhecimento sobre a modelagem matemática é muito importante para desenvolvimento de uma base teórica sólida para formação do engenheiro. Todavia, apenas a modelagem teórica fica limitada à realização de projetos de baixo nível de complexidade (Diniz et al., 2012). Caso fossem ensinadas ferramentas computacionais, poderia resolver projetos com aplicações industriais de alta complexidade. $\mathrm{O}$ Brasil teria possibilidade de desenvolver seus produtos, veículos por exemplos, etc.

O projeto MINI - BAJA é um exemplo de utilização de ferramentas CAD-CAE. Porém, poucos alunos que participam deste projeto e aprendem as ferramentas CAD-CAE. Estes alunos geralmente vão trabalhar em projetos dentro nas indústrias, é disto que o Brasil precisa. Todavia, estes alunos ficam limitados aos conhecimentos que aprenderam sozinhos. Pode ser melhorado com disciplinas ministradas por docente a todos os alunos.

Os professores poderiam investir nesta metodologia de ensino de ensino CAD-CAE. No contrário, o Brasil ficará sempre dependendo de tecnologia das indústrias internacionais.

\subsection{Debates Sobre Formação de Engenheiros Auto- motivos}

Debate entre executivos da indústria automotiva sobre a aplicação de ensaios e simulações no desenvolvimento de produtos, realizado em 2 de agosto de 2006 na Escola Politécnica da Universidade de São Paulo, tiveram as seguintes conclusões (Debate, 2006):

- A indústria mundial de veículos está em crescimento e os novos concorrentes do Brasil - países emergentes e de características semelhantes - investem na especialização da engenharia local;
- Atualmente, o mercado necessita de um número maior de engenheiros especialistas, mas tem dificuldade para encontrá-los;

- A indústria utiliza cada vez mais a simulação, em substituição aos testes físicos, e comprova que a ferramenta reduz o tempo de desenvolvimento de produto e representa economia de gastos;

- O progresso dos softwares e hardwares tem favorecido o crescimento da simulação.

No mesmo debate, foram propostos alguns desafios futuros:

- O Brasil precisa formar, rapidamente, um número maior de engenheiros com conhecimento básico e capacitados para trabalharem no desenvolvimento de produtos e na criação de novos processos. A solução apresentada é o fortalecimento da parceria entre as universidades e o mercado automotivo, para garantir a formação de engenheiros com o perfil adequado. Sugere-se que a indústria ofereça casos reais que possam ser estudados em sala de aula. A relação universidade-empresa poderá, ainda, agilizar o processo de maturação dos engenheiros à condição de experts, conforme o mercado exige;

- A simulação deve ser no futuro a ferramenta mais utilizada, de forma que os testes fiquem concentrados na fase final do projeto, para verificação dos resultados virtuais. No entanto, acredita-se que o volume de testes não diminuirá, porque os produtos serão cada vez mais completos e atenderão requisitos mais exigentes;

- As empresas devem promover a integração entre as equipes de testes físicos e de simulação, além de estimular que a ferramenta virtual seja descentralizada, ou seja, utilizada pelas várias áreas ligadas ao desenvolvimento de produto;

- É importante mudar a imagem de "engenheiros de simulação" para "engenheiros especialistas em desenvolvimento de produto", com o objetivo de aumentar, internamente, a credibilidade da ferramenta virtual;

- As montadoras devem continuar incentivando que os fornecedores utilizem as ferramentas de ensaio e de simulação no desenvolvimento de autopeças. Este debate mostrou, portanto, a emergente necessidade de formação de profissionais para atuação na indústria automotiva.

\subsection{Mercado de Trabalho: $C A D-C A E$}

Diversas indústrias necessitam dos conhecimentos utilizando ferramentas computacionais: 
- Montadoras de veículos, máquinas pesadas, caminhões e máquinas agrícolas;

- Projetos de sistemas estruturais automotivos;

- Industriais de motores, transmissão;

- Industriais de sistemas de suspensão: passiva $\mathrm{x}$ ativa;

- Industriais de sistemas de direção, freio e suspensão.

- Industriais de sistemas de embreagem: mecânica, hidráulica, pneumática;

- Industriais de acústica e vibração veicular;

- Industriais de projeto e análise dinâmica estrutural;

- Industriais de Chassis, carrocerias e uso de CFD;

- Industriais de sistemas de refrigeração e ar condicionado.

\subsection{Ferramentas Computacionais CAD-CAE}

A modelagem assistida por computador tem dois objetivos principais: diminuição de custos e de tempo e o aumento na qualidade do produto final. Essa duas etapas apresentam algumas sub-etapas de desenvolvimento essenciais para o sucesso na realização de cada uma delas (ADAMS/SOLVER, 2005).

Os softwares, em sua maioria, aceitam arquivos criados em outros programas, permitindo assim integrar as diversas áreas de projeto e visualizar o projeto funcionando por completo através de simulação virtual.

\section{CAD - Projeto Assistido Por Computador}

Os sistemas CAD (Computer Aided Design) foram desenvolvidos para substituir o desenho manual, que era demorado e de difícil reaproveitamento. No início, a nova tecnologia foi considerada um substancial avanço, que permitiu a digitalização de grande parte dos projetos e forçou os desenhistas e projetistas a se adequarem a esta nova tecnologia.

A grande versatilidade dos modelos tridimensionais justificou toda a energia gasta na transição entre os dois sistemas (Foggiatto, et al., 2007).

Uma das principais vantagens na utilização dos sistemas CAD está na interatividade com outras áreas. O modelo pode ser utilizado para calcular volume de sólidos, propriedades de massa e momentos de inércia, realizar montagens, verificar interferências, gerar os desenhos de fabricação, simular comportamentos mecânicos, criar prototipagem rápida, usinagem por CNC, entre outros (Foggiatto, et al., 2007).

\section{CAE - Engenharia Assistida por Computador}

CAE é a analise e avaliação do projeto de engenharia usando técnicas computacionais, para calcular a operacionalização e funcionalidade do produto, bem como parâmetros da manufatura muito complexos para se calcular por métodos clássicos (Rehg, 1994).
A Engenharia Assistida por Computador também conhecida como CAE (Computer Aided Engineering) utiliza recursos computacionais para simulação de modelos físicos, englobando a validação, simulação e otimização dos mesmos. É possível analisar como o modelo físico se comporta nas mais diversas condições para que seja verificada a viabilidade da construção do sistema física.

O grande avanço da tecnologia CAE revolucionou a forma de como os projetistas avaliam o desempenho dos seus projetos, poupando assim tempo e dinheiro, uma vez que, em inúmeras situações, é eliminada a necessidade da construção de protótipos. Os softwares CAE permitem que sejam realizados diversos tipos de análises como: estruturais, térmicas, dinâmicas e de fluídos. Por meio dessas análises, uma grande quantidade de cálculos é alcançada em tempo reduzido, resultando em dados de tensão, deformação, velocidade, força, entre outros.

A utilização dos softwares CAE permite que o projetista se concentre por mais tempo nas etapas técnicas e criativas do projeto e o software expõe possíveis erros logo no inicio do projeto, melhorando a qualidade e diminuindo o tempo de entrega dos produtos.

Análise do CAD-CAE

Quando surge a necessidade de analisar uma estrutura, a primeira questão colocada é a classificação dela quanto à geometria, modelo do material constituinte e ações aplicadas. A maneira em que as análises são aplicadas depende das simplificações inerentes a cada tipo de projeto.

As ações sobre as estruturas são, em geral, dinâmicas, devendo ser consideradas as forças de inércia associadas às acelerações que cada um dos componentes fica sujeito. Portanto, é de se esperar que na análise de uma estrutura deve-se obrigatoriamente considerar os efeitos dinâmicos. Contudo, em muitas situações é razoável considerar que as ações são aplicadas de modo suficientemente lento, tornando desprezáveis as forças de inércia. Nestes casos a análise é dita estática (Silva, 2008).

\subsection{MSC. ADAMS}

O MSC. ADAMS é importante para visualização virtual do projeto funcionando (ADAMS/View, 2005). Podemos exportar a dinâmica do sistema e importar os resultados de diversos projetos processados por diversos softwares. Desta forma é possível visualizar o projeto completo: mecânico, hidráulico, pneumático, térmico, controle, aeronáutico, CFD entre outros. Todos sendo simulado integralmente no computador (ADAMS/FULL, 2005). Descrição dos pacotes:

- ADAMS/Solver: É uma ferramenta de análise numérica que resolve equações de movimento para cinemática, estática, dinâmica e simulações; 
- ADAMS/View: É o ambiente de modelagem e simulação que ajuda a resolver problemas de projeto. O ADAMS pode ser usado para construir, simular, e refinar virtualmente modelos de qualquer sistema mecânico;

- ADAMS/Aircraft: Permite criar, classificar e analisar rodas e trem de pouso para montagem completa de aeronaves;

- ADAMS/Car: Permite criar, classificar e analisar suspensão para montagem de veículos;

- ADAMS/Driveline: Permite criar modelos de acionamentos e analisar virtualmente protótipos de subsistemas de acionamento;

- ADAMS/Engine: Permite criar comboios de válvula e, em seguida, analisá-las para compreender a sua elástico-cinemática e comportamento dinâmico;

- ADAMS/Rail: Permite criar modelos ferroviários completos e parametrizados;

- ADAMS/PostProcessor: Permite visualizar os resultados das simulações usando outros produtos ADAMS. Pode-se rodar essa ferramenta diretamente ou dentro de outro produto ADAMS, como o ADAMS/Car ou o ADAMS/Engine.

O ADAMS/Controls é um plug-in que permite ao usuário adicionar sofisticados métodos de controle ao sistema criado. Essa ferramenta possibilita a inclusão do modelo ADAMS a diagramas de blocos desenvolvidos em ferramentas de controle como os softwares MATLAB ou EASY5 (ADAMS / CONTROLS, 2005).

O ADAMS/Controls oferece as opções para simulação do sistema mecânico e do controle inteiramente dentro da ferramenta de controle, simular todo o sistema no ADAMS ou resolver as equações de controle na ferramenta de controle e as equações mecânicas no ADAMS. Esse plug-in também permite ao usuário visualizar os resultados das simulações de forma interativa (ADAMS/CONTROLS, 2005).

Utilizando o ADAMS/Controls pode-se aproveitar o mesmo modelo para os dois projetos (mecânico e de controle). Dessa forma, os processos de testes físicos são simplificados e os riscos (de leis de controle de falhas, por precariedade ou erros na modelagem) são praticamente eliminados, como apresentado no esquema da Figura 2. (ADAMS/CONTROLS, 2005).

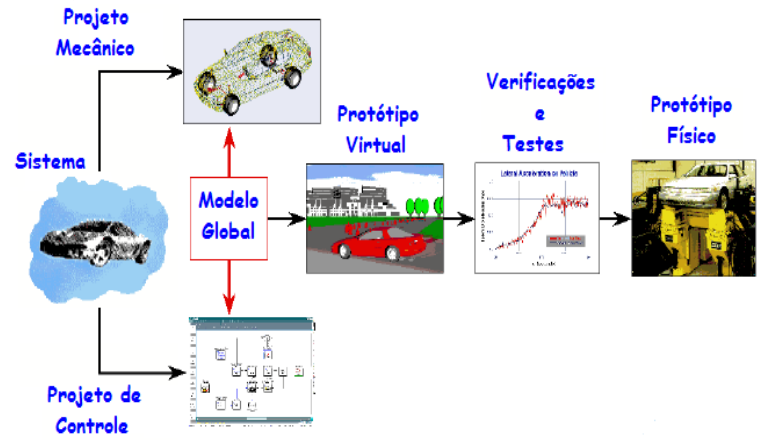

Figura 2. Processo de projeto com ADAMS/Controls.

\subsection{MSC. NASTRAN / PATRAN}

O software MSC/Nastran é um solver utilizado para análise estrutural de desenvolvimento de produtos. Utilizado para otimizar projetos submetidos a esforços, cargas térmicas e fluídos. A compreensão do histórico térmico e do estado térmico podem afetar comportamento estrutural (como guarnições de veículos sobre influência acústica das cabines, fluxo de tensões induzidas ou deformação) é importante para o projetista. Realizar a modelagem manual é praticamente impossível.

O software MSC. Patran é mais utilizado para pré e/ou pós-processamento de Análise de Elementos Finitos (FEA), oferecendo assim modelagem sólida e articulada, também permite a configuração de análise e pós-processamento para vários solucionadores incluindo o MSC. Nastran, Marc, Abaqus, LS-DYNA, ANSYS, e Pam-Crash.

\subsection{NX: CAD-CAE-CAM}

O NX é aplicado a projetos de produtos integrados, simplificando e acelerando o processo de desenvolvimento para engenheiros que precisam criar produtos inovadores em um ambiente colaborativo, representado na figura 3 .

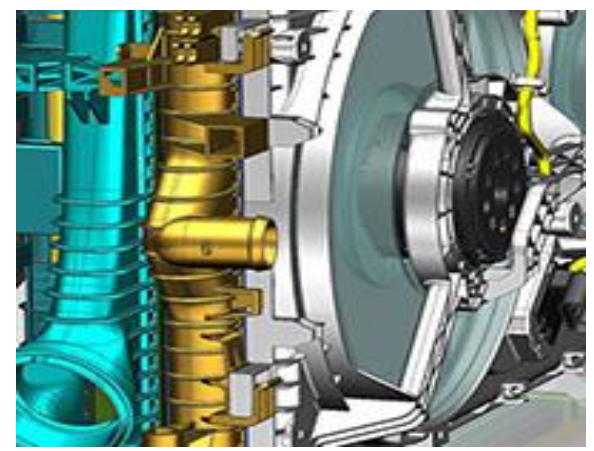

Figura 3 - sistema integrado no ambiente NX

Ao contrário das soluções corporativas fechadas e das soluções com apenas CAD, o NX oferece um nível mais alto de integração entre as disciplinas de desenvolvimento em um ambiente colaborativo e aberto, que podem ser:

- Projeto de produtos;

- Projeto industrial e modelagem;

- Esboço e documentação;

- Projeto eletromecânico;

- Reutilização de conhecimentos;

- Análise visual e validação;

- Aplicativos específicos do processo;

- Ferramentas de produtividade NX.

As soluções do NX aplicado a simulação de engenharia incluem o NX CAE que contém a solução para sistemas dinâmicos e análise estrutural, térmica, 
CFD entre outros analistas avançados de grupos de trabalho e projetistas que precisam proporcionar desempenho de alta qualidade.

O NX CAE integra melhor as classes apresentadas a seguir:

- Modelagem de simulação e visualização de resultados;

- Análise estrutural;

- Análise térmica;

- Simulação de fluxo;

- Análise de movimento;

- Multifísica;

- Otimização da engenharia;

- Dados de simulação e gerenciamento do processo;

- Projeto orientado por simulação;

\section{Materiais e Métodos}

Para a aplicação desta metodologia foi implementado o sistema de um pêndulo invertido rotacional. Neste estudo utilizamos CAD e CAE para realizar o controle de um pêndulo rotativo.

A Universidade Estadual Paulista, no campus de Sorocaba, oferece algumas disciplinas que permitem a modelagem computacional de projetos, que são:

- Projeto e fabricação assistida por computador, com carga horária de 90 horas, sendo destas 60 horas práticas de laboratório;

- Dinâmica de sistemas mecânicos, com 4 horas de laboratórios semanais, no total de 80 horas de carga horária;

- Projeto de mecanismos com grade horária de 80 horas, sendo destas, 15 horas no computador.

- No segundo semestre de 2013, está oferecendo a disciplina Resistência dos Materiais Assistida por Computador. Todo conhecimento da teoria será vista computacionalmente com 60 horas.

Na base didática destas disciplinas são utilizados os softwares NX (motion, advanced Simulation e CAM) e MSC. (da ADAMS, Nastran/Patran e Easy5).

\subsection{Estudo de caso: pêndulo rotativo invertido}

O estudo realizado mostra a implementação dos recursos CAE na engenharia do Brasil. O aluno visualiza o resultado no ambiente virtual, como se fosse jogo.

Considerando o pêndulo invertido rotacional mostrado na Figura 4, observa-se a aplicação de um torque $\tau_{1}$ no "Braço 1". A ligação entre os "Braços 1 e 2" não é atuada, portanto é livre para rotacionar. Os dois braços possuem comprimento $L_{1}$ e $L_{2}$, e massas $m_{1}$ e $m_{2}$ que estão localizadas nos pontos $l_{1}$ e $l_{2}$ respectivamente. Os pontos $l_{1}$ e $l_{2}$ são as respectivas distâncias entre os pontos de rotação dos braços e seus centros de massa (Calkin, 1996) e (Cazzolato, 2007).

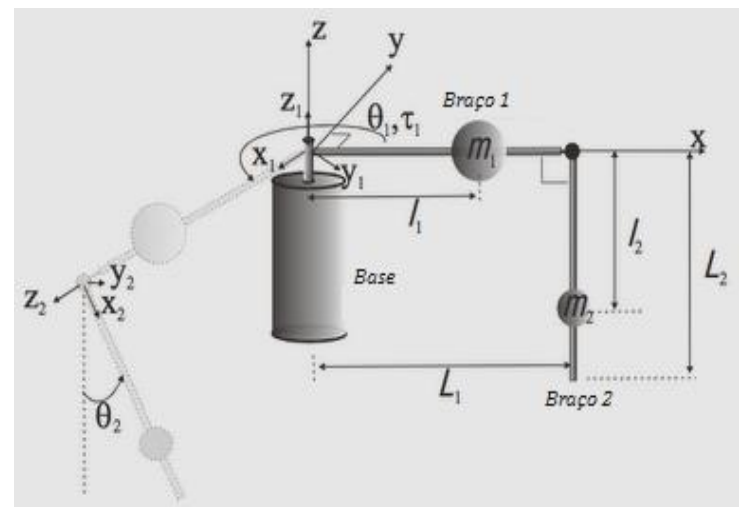

Figura 4. Variáveis do pêndulo invertido rotacional.

Normalmente, faz-se a modelagem matemática de sistema, mas neste estudo como o intuito é apresentar as ferramentas CAE, foi realizado apenas modelagem computacional: CAD-CAE.

\section{Resultados}

O projeto, após ser modelado no ADAMS, teve a sua dinâmica exportada para o software Matlab/Simulink que foi utilizado para testar a semelhança entre os modelos encontrados e a eficiência do controlador projetado.

\subsection{Modelo computacional em espaço de estados}

O modelo computacional foi exporta para o Matlab através da função "ADAMS_sys" que gerou o modelo linear em espaço de estados representado pelas matrizes "ADAMS a", "ADAMS b", "ADAMS_c" e "ADAMS_d". Estas estão apresentadas nas Figuras 5 e 6.

Através dos comandos apresentados nas Figuras 6 e 7 e também das matrizes "ADAMS_a", "ADAMS_b", "ADAMS_c" e "ADAMS_d" foi possível encontrar o modelo computacional na forma de espaço de estados, apresentadas em (1) e (2).

$$
\begin{gathered}
{\left[\begin{array}{l}
\dot{\theta}_{1} \\
\dot{\theta}_{2} \\
\ddot{\theta}_{1} \\
\ddot{\theta}_{2}
\end{array}\right]=\left[\begin{array}{cccc}
0 & 0 & 1 & 0 \\
0 & 0 & 0 & 1 \\
0 & 39,78 & 0 & 0 \\
0 & 132,66 & 0 & 0
\end{array}\right]\left[\begin{array}{l}
\theta_{1} \\
\theta_{2} \\
\dot{\theta}_{1} \\
\dot{\theta}_{2}
\end{array}\right]+\left[\begin{array}{l}
0 \\
0 \\
24,62 \\
36,78
\end{array}\right]\left[\tau_{1}\right]} \\
{\left[\begin{array}{l}
y_{1} \\
y_{2}
\end{array}\right]=\left[\begin{array}{cccc}
1 & 0 & 0 & 0 \\
0 & 1 & 0 & 0
\end{array}\right]\left[\begin{array}{l}
\theta_{1} \\
\theta_{2} \\
\dot{\theta}_{1} \\
\dot{\theta}_{2}
\end{array}\right]}
\end{gathered}
$$




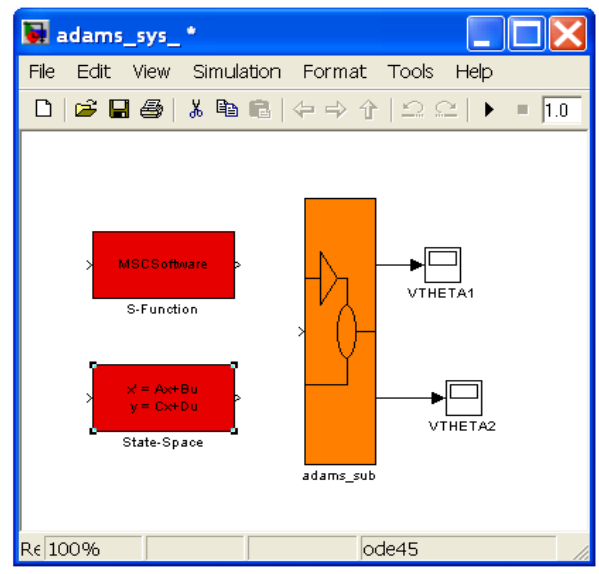

Figura 5. Modelo gerado pela função ADAMS_sys.

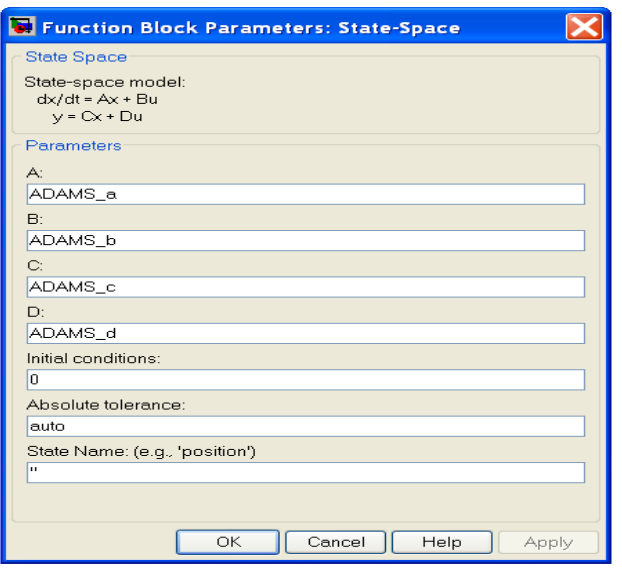

Figura 6. Detalhamento do modelo gerado pela função ADAMS_sys.

A figura 7 representa o resultado do controle realizado computacionamente no ambiente ADAMS.

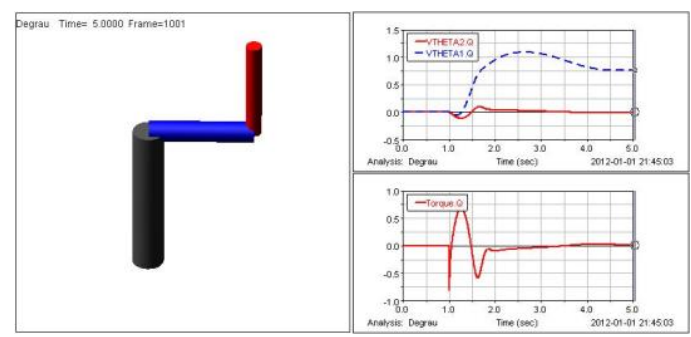

Figura 7. Visualização virtual do controle do pêndulo.

\section{Conclusão}

Este artigo apresentou algumas ferramentas e um exemplo de projeto com modelagem de sistema dinâmico e de controle assistido por computador. É perceptível que torna-se mais interessante analisar o projeto virtualmente e aplicar as técnicas para validação do mesmo. Da mesma forma, podem ser construídos mais projetos, principalmente no âmbito de formação de engenheiros, sobretudo no nível de graduação.
O entusiasmo dos alunos em resolver problemas de engenharia é alto, sobretudo pela aplicação dos conhecimentos adquiridos durante a formação. A implementação de projetos de sistemas dinâmicos, hidráulicos, térmicos, etc. assistidos por computador é fundamental para o crescimento do Brasil. Durante as aulas são resolvidos matematicamente os resultados e mostrado como são válidas as modelagens e por comparação computacional. Após a segurança na metodologia e uma maior prática, são desenvolvidos projetos mais complexos já sem a necessidade de validação matemática.

\section{Referências Bibliográficas}

ADAMS/CONTROLS (2005). Getting Started Using ADAMS/Controls.

ADAMS/FULL (2005). Basic ADAMS Full Simulation Training Guide.

ADAMS/SOLVER (2005). Basic ADAMS/Solver Training Guide.

ADAMS/VIEW (2005). Getting Started Using ADAMS/View.

Calkin, M. G. (1996). Lagrangian and Hamiltonian Mechanics. Cingapura: World Scientific, 1996. DOI: $10.1142 / 3111$

Cazzolato, B. (2007). Derivations of the Dynamics of the Ball and Beam System, Adelaide, 2007.

Diniz, I. S., Colón, D. and et al. (2012). Engenharia Mecânica-Automotiva: Uma Nova Concepção de Projeto Pedagógico. In: VII Congresso Nacional de Engenharia Mecânica (CONEM 2012), 2012, São Luiz do Maranhão. Anais do VII Congresso Nacional de Engenharia Mecânica. Rio de Janeiro, Editora ABCM, 2012.

Debate (2013) sobre ensaios e desenvolvimentos de produtos: Disponível em http://www.automotivapoliusp.org/debate-entre-executivos-daindustria-automotiva-sobre-a-aplicacao-deensaios-e-simulacoes-no-desenvolvimento-deprodutos-3/ . Pesquisa realizada em 24/06/2013.

Foggiatto, J. A., Volpato, N. and Bontorin, A. C. B. (2007). Recomendações para Modelagem em Sistemas CAD-3D. $4^{\circ}$ Congresso Brasileiro de Engenharia de Fabricação, 2007.

Nakamura, K., Yamanaka, R., Matsuda, T. and Harada, T. (2003). Recent developments in asymmetric reduction of ketones with biocatalysts. Tetrahedron. n. 14, 2003.

Silva, J. F. and Diniz, Ivando S. (2008). Modelagem, Simulação e Controle de Um Pêndulo Invertido Auxiliado Por Computador: CAD e CAE. Trabalho de graduação. Universidade Estadual Paulista. Sorocaba, 2008.

Schvarcz, A. F. and Diniz, I. S. (2010). Modelagem, Simulação e Controle de um Sistema Barra e Bola Auxiliado por Computador: CAD E CAE. Universidade Estadual Paulista. Sorocaba. 2010.

Rehg, J. A. 1994. Computer Integrated Manufacturing. New Jersey: Prentice Hall, 1994. 